\title{
Porvenir inmediato y alejado de los niños extraidos por operación cesárea tardía
}

\author{
(Estudio clínico, psiquico y electroencefalográfico)
}

\section{Por B. ROSENVASSER}

Dividiremos este trabajo en tres partes: en la primera analizamos la evolución del criterio quirúrgico en obstetricia y el objeto de esta publicación; en el segundo, la mortandad y neomortandad fetal por operación ce. sárea en el Instituto de Maternidad y Asistencia Social *Profesor U. Fernán. dez», que dirige el Profesor Manuel Luis Pérez, de 1942 a 1949, y en el terzero el porvenir (alejado) de los niños extraídos por operación cesárea tar. dia.

\section{PRIMERA PARTE}

De Lee y Greenhill (1) definen a la operación cesárea como «el acto quirúrgico por medio del cual se extrae del útero al feto, a través de una incisión realizada en la pared abdominal. Este término no se aplica a la extracción felal despues de una ruptura ute: rina, ni tampoco a las operaciones que se ejecutan con motivo de un embara zo ectópicon. Excluímos también a la cesárea vaginal.

Relativo a su técnica, cabe decir. que hasta hace pocos años se practi. caban las siguientes intervenciones: a) Cesárea transperitoneal (corporal o segmentaria).

b) Cesárea extraperitoneal (pura 0 por artificio).

c) Operación de Porro.

d) Operación de Gottschallk-Portes.

Con el advenimiento de las sulfodrogas $y$ de los antibióticos, la tendencia actual es la realización de la operación cesćrea segmentaria transperitoseal. con el abandono de las demús técnicas por la mayoría de los auto. res.

Su primera Indicación (exclusiva en sus albores), fue la estrechez pelviana, actualmente y siguiendo a Infantozzi (2) consideraremos como:

\section{Indicaciones Absolutas:}

1) Estrochez pélvica:

a) Estrechez en el estrecho superior: A) Pelvis plana pura: P. P. menor de siete centímetros; B) Pelvis plana y generalmente estrechada: P. P. M. menor de ocho centímetros; C) Pelvis generalmente estrechada: P. P. M. menor de nueve centimetros.

b) Pelvis canaliculada: diámetro útil menor de siete centímetros. 


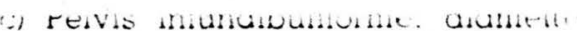

bi-isquiático, menor de siete centimetros.

Nos referimos en casos con fetos a térmu:iv y de dimensiones normales o mayores

2) Tumores previos: Solidos o liqui. dos que sean irreductibles.

3) Placenta previa oclusiva centro to tal sangrante.

4) Anomalias del cuello uterino: $\mathrm{Ca}$ sos de atresia. cuello escleroso o cicatrizal que no permite la dilatación a la cervicotomía. Agregaremos por nuestra parte:

5) Distocia comploja.

Indicaciones rolativas:

1) Placenta previa; 2) Desprendimiento normoplacentario; 3) Distocia contráctil; 4) Estrechez relativa de pelvis: 5) Presentaciones viciosas (tronco, frente, pelviana); 6) Diabetes materna; 7) Incompatibilidad por factor Rh; 8) Gestosis; 9) Algunas cardiopatias; 10) Embarazo y carcinoma del cuello uterino (histerectomía); 11) Procidencia o procúbito del cordón: 12) Fracaso de otra intervención (tórceps, inducción), etc.

Luégo de referirnos a la tócnica y a las indicaclones de la operación cesá rea vamos a analizar ahora como ha evolucionado el criterio quirúrgico en lo relativo a momento de realización de la intervención.

Siguiendo a Manuel Luis Pérez (3). consideraremos como cesdreas tardías aquéllas que se practican con más de 10 horas de trabajo de parto o más de 12 horas de bolsa rota.

Para comprender el objelo de esta publicación debemos hacer una rese ña de los criterios mantenidos en las distintas épocas por diferentes tocólo. gos, parq decidirse a la histerolomía

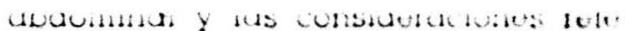

rentes a los fetos obtenidos por dichos procedimientos quirúrgicos.

Lo primero que debemos resaltar es la enorme influencia que ha tenido en la obstetricia (al igual que en las res. tantes especialidades médicas), el des. cubrimiento de productos quimioterá picos y antibióticos, es asi que proble. mas planteados como insalvables, , gracias al uso de sulfodrogas, penici. lina, estreptomicina, etc., han termina. do con resultados plenamente satistactorios.

Hay autores, tales como A. A. Lo bedev (4), Brandberg (5), W. Bromberger (6), H. C. Miller (7) y S. A. Selitzky (8), quienes estudiando las cesáreas y los tetos obtenidos no consideran la duración del parto, es decir, no valoran la importancia del parto de prueba.

Otros analizan la práctica del parto de prueba y se muestran opuestos a la recalización del miamo. Brander (9) en. contró 72 casos de lesiones intracraneanas confirmadas post-mortem en niños extraídos por cesárea abdomlnal. Halló tambièn niños con defectos de la inteligencia, convulsiones epileptiformes y paresias espásticas que habian presentado injurias intractanea. nes durante el parto: antro éstas, ubi. ca al parto prolongado. G. Monthaupt (10), de 90 nifios extraídos por cesárea y seguldos después, durante varíos años, encontró 10 con retardo psíquico $y$ en el desarrollo. Los 10 provenian de partos proiongados. También Alonso Romeo y C. S. de Alonso (11); V. Lane (12); Hideo Yagi (13); K. Hollosi (14); E. M. Bridge (15); C. Ramírez y Muilca Lorca (16); W. D. Belnap y C. S. Beck (17) y otros, abogan por la ewpresión dol parto do prueba o por lo menos reducelon do su duractor. 
El examen de otros trabaios nos pe:mite concretar la existencia de numerosos tocólogos en disidencia con el criterio de la práctica de la operación cesárea precoz. En las Terceras Jornadas Chilenas de Obstetricia y Ginecología, V. M. Gazitua, J. León, M. Ro driguez López. F. Rodriguez y R. Garcia Valenzuela, se declararon partidarios del parto de prueba por considerarlo innocuo para el binomio madre-hijo, en oposición al concepto de los relatores C. Ramirez y Mujica Lorca, ya citados. L. M. Dantuono (16). considera que el parto de prueba debe prolongarse durante 30 horas. H. M. Keith y M. A. Norval (19), descartan las dificultades del parto (lógicamente bien solucionadas), y el largo trabajo de parto como factores perjudiciales para el feto. Iguai criterio sustentan $R$. Landesman (20). J. K. Kuhn (21), R. Pad dok (22) y $\mathrm{H}$. Laenger (23).

Vemos, pues, que se discute aún la práctica del parto de prueba, pues si bien hay escuelas que lo rechazan, otras lo consideran indispensable.

Boero E. A. (24), ha publicado diversos trabajos sobre cesáreas tardias refiriéndose solamente a su parte técni. ca. Comprendemos que habiendo sido escritos mucho antes de la éra antibiótica, exigia el cumplimiento de ciertas condiciones para la práctica de la operación cesárea; tales eran: huevo integro y por lo menos 72 horas sin que se le hubiesen practicado tactos vaginales.

En las épocas actuales, especial. mente en nuestro servicio en el que prima un criterio ecléctico en cuanto a indicaciones de la operación cesárea (no intervencionista como ocurre en las escuelas norteamericanas, ni demasiado expeciante como ocurre en otras); considerando que el uso de los antibióticos permite la realización de tactos vaginales (logicamente hechos con la mayor asepsia posible) y con iemporiza el parto de prueba de va. rias horas de duración. ha hecho car ne en nosotros digamos, Ia realizacion de la cesárea tardía, es decir, cuando ha iracasado el parto do prueba o cuando ésto debo ser interrumpido por alteración de los latidos fetales u otra causa. En tal sentido, citaremos los trabajos de Manuel Luis Pérez y Ramón Echeverria (25), Julio Bazán, F. A. Uranga Imaz y Schiavo (26), Silvestre Sala (27), Juan León (28), F. Rodríguez y G. J. Roubillard (29). Así E. Bayona y R. M. Gori (30), concluyen que el uso de los antibióticos ha disminuido enormemente la morbi-mortalidad materna y que al permitir un parto de prueba prolongado, muchos casos de estre. chez relativa de pelvis terminaron su parto por vias naturales, disminuyendo asi el número de cesáreas practicadas.

Pero se nos plantea el siguiente interrogante: ¿El niño en los casos en que ha habido "lucha" de varias horas, no presentará trastomos a posteriori? ¿Estará indemne de trastornos encefálicos de toda indole? De aquí deducimos el objetivo que guía este trabajo: Valorar fundamentalmente los distintos factores que intervienen en la operacián cesárea, especialmente lo relativo al parto de prueba, para guiar nuestra conducta a una mayor seguridad en el pronóstico del niño por operación cesárea; decidir si es la con. ducta precoz o la tardía la que se de. be seguir como más favorable. 


\section{SEGUNDA PARTE}

\section{Porvenir inmediato de $l 08$ niños} extraídos por cesćrea tardía

El ideal de la obstetricia -especialmente on los tiempos modernos-- es obtener una criatura que nazca viva y sma, exenta de malformaciones con. genitas $y$ que esa eventualidad no periudique el organismo materno. El mejor conocimiento de la fisiología y de la patología obstétrica, el mejor y más correcto uso de diversas drogas (derivados o extractos hipofisarios, antiespasmódicos, analgésicos. sedantes, etc.) y el advenimiento de las sulfogrogas $y$ de los antibióticos ha mejorado enormemente el pronóstico de la madre y del niñc, especialmente desde el punto de vista materno, ya que aproximadamente el $4 \%$ de los niños mueren en el parto o por hechos vinculados al mismo. Debemos calcular en casi 100.000 , el número de criaturas que anualmente mueren al nacer 0 en el primer día de vida extra. uterina. Un gran porcentaje de niños sufren, al nacer, lesiones encelálicas más o menos importantes, aun en los partos normales. Es asi que en las au topsias de recién nacidos es trecuente observar hemorragias (puntiformes 0 más extensais) en el encéfalo, en los grandes ganglios, senos $y$ suturas. Es innegable que tales extravasaciones sanguineas deben deiar cicatrices en las distintas estructuras cerebrales, de diferente tamaño e importancia según su cantidad y localización; estas cicatrices pueden explicar algunos siste. mas cerebrales que aparecen en épo. cas ulteriores de la vida.

Antes de seguir adelante, debemós aclarar varios conceptos. Existe una anarquia muy grande respecto a las detiniciones de mortalidad y neomortandad fetal: de acuerdo con Josué A. Beruti (31) llamaremos: mortalldad in. fantil o neomortalidad. la muerte del neonato dentro del primer año de vi. da: mortalidad o mortalidad neonatal precoz. la que se refiere únicamente a los primeros dias de vida; hobdoma. daria o primohebdomadaria hasta los siete dias, inclusive; decadaria o primodecadaria hasta los 10 dias, inclusive, y total o nosocomial a la que co rresponde al período de internación de la enferma en la clinica; entre ésta y la decadaria hay poca diferencia y como ésta corresponde a la época en que los neonetos recuperan el peso y durante la cual la mayoría de ellos mueren por causas imputables al parto, será ella la que tendremos en cuenta.

J. P. Greenhill (32), aconseja para mejorar el porvenir inmediato del nino: cuidados pre-natales, prevención y tratamiento de la prematurez, preven. ción de las injurias del parto, prevención de las infecciones (especialmente neumonía y siflilis), prevención y precoz tratamiento de las toxemias gravidicas y diagnóstico pre-parto y tralamiento rápido de la eritroblastosis.

J. Lascano y E. S. Halac (33), V. Gazitúa Guzmán (34), N. Palacios Costa, F. Peiretti y R. Salomone Allievi (35), A. Peralta Ramos y R. Dubrovsky (36), 1. A. Gabastou (37), E. Argons (38), M. L. Pérez y R. Echevartía (39) y M. L. Pérez y M R. de Ginocchio (40), en distintos trabajos esbozan -cuando no lo expresan trancamente- que el trebajo de parto prolongado alimenta el porcentaje de mortinatalldad.

Tres son las causas más frecuentes de mortandad nosocomial Intantil: as. 
7) Las' intervenciones practicadas con sufrimiento fetal se acompañaron de una mortandad nosocomial mucho mayor que aquéllas en que no había existido sufrimiento fetal.

8) En contraposición a lo expresado por la mayor parte de los autores, el largo trabajo de parto o la existencia de bolsa rola de más de 12 horas de duración no cumentó el número de fotos natimortos; por el contrario, las cesćreas precoces se acompañaron siempre de mayor porcentaje de fetos natimortos que las cesćreas tardías; posiblemente influyó en estas cliras que la placenta previa y el desprendimiento normoplacentario fueron indicaciones de cesáreas precoces y muchas veces causa de la muerte fetal.

$P$ ir consigulente, según nuestras ob servaciones, el largo trabajo do parto - totalmente innocuo para el parvenir inmediato del niño.

Veremos en el próximo capítulo, la Influencia de dicho lango trabajo de parto sabre el porvenir alojado dol niño.

(Continuaród)

\section{PARA PROPAGaNdA EN ESTA REVISTA}

entenderse con el Administrador:

\section{JUAN N. BAQUERO}

en su nueva dirección:

Calle 16, No 7.91 (segundo piso) Oficina No 4.

teléfono 23-901

Apartado número 276.

BOGOTA - COLOMBIA 